\title{
Effects of probiotics supplementation on placental microbiome in healthy women undergoing spontaneous delivery
}

\section{Ping Yang}

The First Affiliated Hospital of Jinan University

\section{Zhe Li}

The Third Affiliated Hospital of Sun Yat-Sen University

\section{TYE KIAN DENG}

The First Affiliated Hospital of Jinan University

\section{Tong Lu}

Shenzhen Long Hua District Central Hospital

\section{Yuyi Chen}

The First Affiliated Hospital of Jinan University

\section{Zonglin He}

The First Affiliated Hospital of Jinan University

\section{Juan Zhou}

The First Affiliated Hospital of Jinan University

\section{Xiaomin Xiao ( $\nabla$ xiaoxiaomin55@yeah.net)}

The First Affiliated Hospital of Jinan University

\section{Research Article}

Keywords: probiotic, full term pregnancy, 16S rRNA sequencing, interaction network, placental microbiota

Posted Date: April 21st, 2021

DOI: https://doi.org/10.21203/rs.3.rs-418396/v1

License: (c) (1) This work is licensed under a Creative Commons Attribution 4.0 International License. Read Full License 
Effects of probiotics supplementation on placental microbiome in healthy women undergoing spontaneous delivery

Ping Yang ${ }^{1}$, Zhe $\mathrm{Li}^{2}$, TYE KIAN DENG ${ }^{1}$, Tong $\mathrm{Lu}^{3}$, Yuyi Chen ${ }^{1}$, Zonglin $\mathrm{He}^{4}$, Juan Zhou ${ }^{1}$, Xiaomin Xiao ${ }^{1 \#}$

${ }^{1}$ Department of Obstetrics and Gynecology, The First Affiliated Hospital of Jinan University, Guangzhou, China.

${ }^{2}$ Department of Obstetrics and Gynecology, The Third Affiliated Hospital of Sun Yat-Sen University, Guangzhou, China.

${ }^{3}$ Department of Otolaryngology, Shenzhen Long Hua District Central Hospital, Shenzhen, China, ${ }^{4}$ International School, Jinan University.

${ }^{\#}$ Correspondence author

Xiaomin Xiao

xiaoxiaomin55@yeah.net

\section{Abstract}

Purpose to investigate the effect of orally supplemented probiotic on term placental microbiota and provide possible evidences for clinical management of pregnant women. Methods A population-based cohort of specimens were collected from 37 healthy nulliparous pregnant women who underwent systemic examination. The pregnant women were divided randomly into probiotics group and control group. We collected placental specimens during spontaneous delivery at term. The placental samples were taken for analyzing microbiome with the 16S rRNA amplicon sequencing of V4 region. Results There were no significant differences between two groups in clinical characteristics. The placental microbiota from normal vaginal delivery were composed of Bacteroides, Lactobacillus and Ralstonia. The relative abundant of Prevotella_9, Faecalibacterium, and Escherichia_Shigella etc. in probiotic group was significantly higher than that control group $(p<0.05)$. Probiotics supplementation could affect the network structure of placental microbiota. Conclusions The characteristics of the placental microbiome changed after probiotics supplementation and the network structure of interaction tended to be loose. The probiotic supplementation may be useful in regulating the interaction network of placental microbiota.

Keywords: probiotic, full-term pregnancy, 16S rRNA sequencing, interaction network, placental microbiota

\section{Introduction}

Probiotic supplements are widely promoted as "over-the-counter medicines" and can be taken as an auxiliary medicine for human health by modifying the existing microbiota community. Furthermore, it is gradually applied to pregnant women. Therefore, many pregnant women can voluntarily supplement some probiotics during pregnancy, such as golden bifid, acidophilus milk and so on. The use of probiotics in the United States and Canada ranges from 1.3\% to 3.6\%, whereas the probability of using probiotics in pregnant women in Netherlands has risen to $13.7 \%$ [1]. Meanwhile, the International Scientific Association for Probiotics and Prebiotics (ISSAPP) defined the probiotics as an active microorganism, the supplementation of appropriate amounts of which may have a positive effect on human metabolic function and health, where Lactobacillus and Bifidobacterium are widely used[2]. Furthermore, it is used in obstetrics to prevent premature birth and reduce the occurrence of gestational diabetes mellitus. Studies have shown that pregnant women who take probiotics during the first trimester can reduce the diagnostic rate of gestational diabetes mellitus by $60 \%[3]$. What's more, The risk of spontaneous preterm birth is lower in pregnant women who often take probiotics[4]. An 
improved maternal microbiota is likely to provide the beneficial microbes through either direct colonization of the neonatal gastro-intestinal tract or indirect effect on the succession of indigenous intestinal bacteria[5]. Despite the popularity of Golden Bifid in the application in the obstetrics, questions remained: how would the intake of Golden Bifid affect the placental microbiota, and subsequently influence the perinatal outcomes in women with normal pregnancy?

Therefore, this study aimed to investigate the effect of orally supplemented probiotic on term placental microbiota and provide possible evidences for clinical management of pregnant women.

\section{Methods}

\section{Research materials}

\section{Participants}

According to the principle of informed consent, 37 patients with normal physiological pregnancy who planned to give birth in the First Affiliated Hospital of Jinan University (Guangzhou, Guangdong, China) and met the inclusion criteria were recruited. All the participants were Chinese, conceive naturally, singleton pregnancy, nulliparous and under 35 years old. Pregnant women with complications and cesarean section were excluded in the follow-up period, and finally 25 pregnant women with spontaneous delivery were enrolled (Figure 1). The probiotic group (10 case were supplemented with Golden Bifid through the oral cavity, and they took 2 tablets twice a day (2g/d) from 32 weeks of gestation until delivery. Each tablet contains $0.5 * 10^{7} / \mathrm{CFU}$ of live Bifidobacterium, $0.5 * 10^{6} / \mathrm{CFU}$ of Lactobacillus and Streptococcus. Yet the control group took no pills. All the participants were kept in touch with us and their dietary habits were relatively consistent. The antibiotics or other foods contained probiotics during the study were not allowed.

In this study, after consent was obtained, collected and analyzed clinical characteristics from participants included age, height, weight before delivery, BMI before delivery, Apgar score, birth weight, head circumference and length of newborn. Placental specimens were collected under clean and sterile conditions within 30 minutes of spontaneous delivery at term. The placental surfaces were excised and discarded, and the area of necrosis and calcification of the maternal surface of the term placenta were avoided. In the fetal face of the placenta, four $1 * 1 * 1 \mathrm{~cm}$ cuboidal sections are circumferentially excised from separate areas of the placenta, each located $3 \mathrm{~cm}$ from the cord insertion site. Finally, placental specimens were immediately placed in dedicated specimen box, transported to the laboratory within half an hour, and then stored at $-80^{\circ} \mathrm{C}$ until DNA extraction.

\section{High-throughput sequencing}

After extraction of the DNA of microorganisms in specimens, we used the IonS5TM XL sequencing platform to sequence the 16srRNA V4 regions. Cutadapt

(V1.9.1,http://cutadapt.readthedocs.io/en/stable/) was used to perform low-quality partial shearing on the reads, then the sample data were split from the obtained reads according to Barcode; we then cut off the initial data of Barcode and primer sequences to obtain the original data. The Reads obtained were further processed to remove the chimera sequence, and the Reads sequence is passed[6]. We then compared the chimeric sequences with the database (Gold database,

http://drive5.com/uchime/uchime download.html) and finally removed the chimeric sequences[7], and got the final valid data (Clean Reads). All procedures strictly followed the product manual.

\section{OTU clustering and annotation:}

The Uparse software (Uparse v7.0.1001, http://drive5.com/uparse/)[8] was used to cluster all Clean Reads from all samples. By default, the sequences are clustered into Operational Taxonomic Units (OTUs) with 97\% identity. At the same time the the most frequently occurring sequence of OTUs was 
selected as representative sequence according to its algorithm principle. Specimen annotation of OTUs representative sequences and species annotation analysis (set threshold of 0.8-1) were performed using the Mothur method and the SSUrRNA database of SILVA （ http://www.arb-silva.de/） [9, 10], Taxonomic information was obtained and the community composition of each sample was counted at each taxonomic level (such as kingdom, phylum, class, order, family, genus and species). Fast multi-sequence alignment was performed using MUSCLE[11] (Version 3.8.31,

http://www.drive5.com/muscle/), where a systematic relationship of all OTUs representative sequences was obtained.

\section{Statistical analysis}

Clinical characteristics processing was performed using SPSS19.0, $t$ test was used to determine the significance of continuous metadata for study subjects. The basic data were summarized by means of descriptive statistics, including proportion, and mean \pm standard deviation (SD). The LDA Effect Size (LEfSe: Linear Discriminant Analysis Effect Size) was used to detect significant features, which differentiate groups and rank these features by effect size (http://huttenhower.sph.harvard.edu/galaxy/). The threshold used on the logarithmic linear discriminate analysis score for discriminative feature was 3.0. The Spearman analysis was used to analyze the correlation of interaction network structure. $P<0.05$ was considered statistically significant. The R language and Origin8.0 were applied to statistical plotting.

\section{Results}

\section{Clinical characteristics of the study subjects}

For this study, the clinical and demographic characteristics of pregnant women who had a term delivery are displayed in Table 1. There are no significant differences on dietary habits, age, height, weight before delivery, BMI before delivery and birth weight of newborn between two groups.

\section{The feature of full-term placental microbiota in the two groups at the genus level}

At the genus level, the distribution of microbial communities with operational taxonomic units (OTUs) contents above 0.01 in the placental microbiota based on 16S rRNA gene deep sequencing analysis was illustrated in the Figure 2.

By screening the OTUs-table, there are 12 genera whose relative abundance of 0.01 or higher (i.e. high-abundant microbial communities) in term placental microbiome (Figure 2). Among them, 8 genera were detected in control group (UPP), namely Streptococcus, Bifidobacterium, Lactobacillus, Bacteroides, Blautia, Acinetobacter, Ralstonia and unidentified_Chloroplast. Yet, the relative abundance of the OTUs more than 0.01 only accounted for $31 \%$ (Table 2). The remainder represents low-abundant bacteria or unidentified genus. However, in addition to the above-mentioned genus, Escherichia-Shigella, Prevotella_9, Faecalibacterium, and Alistipes were detected in probiotic group (PPP), and the relative abundance of the OTUs more than 0.01 only accounted for $35 \%$ (Table 2). As shown is Table 3, the OTUs contents of the high-abundant microbial communities of placental microbiome were showed.

\section{The difference of placental microbiome between the two groups}

Applying LEfse to identify significant features of the full-term placental microbiome of two groups. In Figure 3a, there were significant differences about full-term placental microbiome between the control group (UPP) and the probiotic group (PPP) $(p<0.05)$. Namely, the relative abundance of placental microbiome in probiotic group were significantly higher than those in control group, and including Prevotella_9, Faecalibacterium, Escherichia_Shigella, Blautia, Parabacteroides, Veillonella, Fusicatenibacter, Lachnoclostridium and Ruminococcus_gnavus_group (Table 4). Moreover, those 
genera were all labeled in Figure 3b. In all panels, red regions and dots are used to designate cases with probiotic group, and green to designate control group.

\section{The interaction network structure of placental microbiome in the two group}

In this study, Figure 4 presented the interaction network structure between the placental microbiome in the control group, with significant statistical support $(r \geq \pm 0.6, p<0.05)$. In probiotic group, we also displayed with statistical significance the interaction network structure between placental microbiome (Figure 5). As shown, we observed that each genus is related to one or more genera (i.e. high-density correlation). In the control group, the interaction network of the placental microbiome was close, with the clustering coefficient of 0.606 (Table 5). However, the interaction network structure of the probiotics group became loose, with the clustering coefficient of 0.469 (Table 5).

What is more, in control group, the number of significant correlations between three genera (i.e. Lactobacillus, Streptococcus and Bifidobacterium) and other genera were 5, 38 and 24, respectively (Table 6). In probiotic group, the number of significant correlations between three genera and other genera were 4, 2 and 8, respectively (Table 6).

\section{Discussion}

In this study, the probiotic supplementation showed that there was no adverse effect on pregnant women and fetus. The pregnant women in the control group and probiotics group were full-term vaginal delivery, and there was no significant difference in fetal growth and development indicators between the two groups.

Microbiota are the earliest life form on the earth. Without microbiota, human beings will not be able to survive healthy. The human body is a "super organism" composed of its own cells and microbial cells. Similarly, the fetus has lived in a micro-ecological environment composed of commensal bacteria. In particular, the placenta, which provides nutrition for the fetus, contains a unique microbiota. Our results show that the placental tissue of normal full-term healthy pregnant women contains symbiotic bacteria. These symbiotic bacteria have the characteristics of various species and low relative abundance, the top three genera were Bacteroides, Lactobacillus and Ralstonia (Table3). At present, with the development of high-throughput sequencing and the increasing research of placenta by related scholars, it has been confirmed that there was microbiota in umbilical cord blood, amniotic fluid, and placental tissue from healthy maternal and fetal[12-15]. In addition, many researchers reported that the placental microbiome is crucial for the maintenance of normal pregnancy and offspring's growth and development[16-19]. Full-term vaginal delivery is the basic law of pregnant women's reproduction, and also in line with the normal process of pregnancy and delivery. Thus, the placental tissue in this study is all vaginal delivery, and the normal microbiota in the vagina may affect the placental microbiome, which is the highlight of this project, that is to explore the characteristics of placental microbiome under normal physiological conditions. Meanwhile, the pregnant women included in this study had no symptoms of vaginitis, such as pruritus, abnormal leucorrhea, etc. There are many hypotheses about the origin of placental microbiota, such as oral cavity, vagina and gut of pregnant women, but the specific path is still in the exploration stage. Thought the specific source pathway of the placental microbiota is still unknown, the connection and circulation among different bacteriallll banks has been reported and proven[20,21]. The similarities between the human oral and placental epithelia and the microbial communities have been suggested to explain this phenomenon[15, 22]. What's more, scholars have found that the placental microbiome can also affect the establishment of gut microbiome, and thus influence the growth and development, as well as susceptibility to immune diseases and neurological diseases[19, 23]. 
Through LEfse analysis in this study, we found that probiotics supplementation can increase the relative abundance of Prevotella_9, Faecalibacterium, Escherichia_Shigella, Blautia, Parabacteroides, Veillonella, Fusicatenibacter, Lachnoclostridium and Ruminococcus_gnavus_group. in placenta microbiome (Figure 3/Table 4). However, there was no difference in the relative abundance of Lactobacillus, Streptococcus and Bifidobacterium between the control group and probiotics group. The reason for the above results may be that the dosage of probiotics supplement is too small or the lack of sample size fails to make significant difference between the two groups. What's more, the above-mentioned increased bacteria may play a potential role in placental function and the growth and development of fetus and infant.

As shown in Figure 4/5, the interaction network structure of the control group was closely connected, that is, there was a significant correlation between bacteria. Among them, Streptococcus may be dominant in placental microbiome. However, in the probiotic group, the interaction network structure had tended to be loose. Furthermore, Bifidobacterium maybe dominant in the placental microbiome. Streptococcus is Gram-positive, aerobe or facultative anaerobe. Most of them are commensal bacteria. In the placental microbiome of control group, it plays a synergistic role with many bacteria through positive correlation, so as to maintain the physiological function of placenta and the growth and development of fetus (Table 6). However, Bifidobacterium is Gram-positive and anaerobic. It, as a kind of physiological beneficial bacteria, provides nutrition and immune regulation for the body. In the probiotics group, Bifidobacterium has a negative correlation with other bacteria and mainly plays an antagonistic role (Table 6). Meanwhile, it can inhibit the growth of harmful bacteria, resist the infection of pathogens, synthesize vitamins needed by human body, and its metabolites can regulate $\mathrm{pH}$, activate the immune system and play an important role[24]. Thus, probiotics supplementation made the structure of the interaction network structure of the placental microbiome tend to be loose, and enhance the effect of Bifidobacterium, which may provide a theoretical basis for drug intervention of placental diseases.

Golden Bifid, live combined Bifidobacterium and lactobacillus tablets, is a way to supplement probiotics during pregnancy. Probiotics have long been renowned for its benefits to human health[25], especially Lactobacillus and Bifidobacterium, which are closely related to reduce body weight gain, fat mass development, inflammation, and hepatic steatosis or to improve glucose homeostasis[26]. Research suggested that probiotic supplementation could change the structure of gut microbiome, by promoting the growth of beneficial commensal bacteria and inhibiting the growth of harmful commensal bacteria[27]. In this study by Zhou et al.[28], the intervention of probiotics can increase the beneficial anaerobic bacteria and maintain the stability of gut microbiome in the mice model. Meanwhile, the application of probiotics in obstetrics is also very extensive, which may play a positive role in the prevention of premature delivery and gestational diabetes[3, 4, 29]. In addition, Jeanjacques et al[30] related scholars reported that probiotics supplementation during pregnancy maybe not have adverse effects on cesarean section, neonatal birth weight and gestational age. This is also consistent with our results.

Overall, the placental tissue of normal full-term healthy pregnant women contains symbiotic bacteria. These symbiotic bacteria have the characteristics of various species and low relative abundance. probiotics supplementation can increase the relative abundance of Prevotella_9, Faecalibacterium, Escherichia_Shigella, Blautia, Parabacteroides, Veillonella, Fusicatenibacter, Lachnoclostridium and Ruminococcus_gnavus_group. What's more, probiotics supplementation maybe significantly modify the interaction network structure of full-term placental microbiome. Whether this change can lead to 
better perinatal outcomes are not aware. However, the effect of probiotics supplementation may provide a theoretical basis for drug intervention of placental diseases. Meanwhile, other researchers of our research group also collected oral cavity, gut and vaginal secretion of the same subjects. Then this study is mainly aimed at the analysis of placental microbiome. In the future, we may analyze the flora structure of different parts.

\section{Limitations}

There are some limitations to our study. First, the choosing of the women undergoing spontaneous delivery guaranteed the normal physiological integrity, but we cannot exclude the impact of bacterial transfer during vaginal delivery. Second, the limited amount of subject may eventually affect the reliability of our results. Third, we failed to collect indicators of growth and development of infants after delivery.

\section{Conclusions}

Though the above limitations, our study demonstrated that the placental tissue of normal full-term healthy pregnant women have the characteristics of various species and low relative abundance. probiotics supplementation made the structure of the interaction network structure of the placental microbiome tend to be loose, and enhance the effect of Bifidobacterium. Thus, the probiotic supplementation may be useful in regulating the interaction network of placental microbiome. the effect of probiotics supplementation may provide a theoretical basis for drug intervention of placental diseases.

\section{Abbreviations}

ISAPP: International Scientific Association for Probiotics and Prebiotics; OTUs: Operational Taxonomic Units; LEfSe: LDA Effect Size; UPP: control group; PPP: probiotic group

\section{Acknowledgements}

We gratefully acknowledge the following investigators who participated in this multicenter study: Zhe Li, TYE KIAN DENG, Yuyi Chen, Tong Lu, Zonglin He, Juan Zhou, Xiaomin Xiao.

\section{Author contributions}

XM. X. and Z.L. conceived and designed the study, P.Y., Z. L., and TYE KIAN DENG performed placenta tissue sampling, P.Y., Z. L., J.Z. and TYE KIAN DENG traced the subjects, P.Y. and Z.L. performed experiments, P.Y. and YY.C. analyzed the data, P.Y., T.L., and ZL. H. wrote the article. All authors made comments on the manuscript. All authors read and approved the final manuscript.

\section{Funding}

This research was funded by the National Natural Science Foundation of China (81771664).

\section{Availability of data and materials}

The datasets generated and/or analyzed during the current study are not publicly available but are available from the corresponding author on reasonable request.

\section{Ethics approval and consent to participate}

According to the ethical principles of the methods for ethical review of biomedical research involving human beings (Trial Implementation (2007), SFDA quality management standards for clinical trials of drugs (2003), provisions for clinical trials of medical devices (2004), WMA declaration of Helsinki and CIOMS international ethical guidelines for biomedical research of human beings and the review of the ethics committee. The contents and methods of the study conform to the medical ethics and requirements, and finally approved. We carry out clinical studies in strict accordance with the scheme approved by the ethics committee and informed consent to protect the health and rights of the subjects. The study project was authorized by the Institutional Review Board (IRB) for Human 
Subjects Research at The First Affiliated Hospital of Jinan University and the approval number was: 2019-011.

\section{Consent for publication}

Not applicable.

\section{Competing interests}

No author has any potential conflict of interest statement

\section{Author details}

${ }^{1}$ Department of Obstetrics and Gynecology, The First Affiliated Hospital of Jinan University, Guangzhou, China. ${ }^{2}$ Department of Obstetrics and Gynecology, The Third Affiliated Hospital of Sun Yat-Sen University, Guangzhou, China. ${ }^{3}$ Department of Otolaryngology, Shenzhen Long Hua District Central Hospital, Shenzhen, China, ${ }^{4}$ International School, Jinan University.

\section{References}

1. Rutten N, Van der Gugten A, Uiterwaal C, Vlieger A, Rijkers G, Van der Ent K. Maternal use of probiotics during pregnancy and effects on their offspring's health in an unselected population. European Journal of Pediatrics. 2016;175(2):229-35. doi:10.1007/s00431-015-2618-1.

2. Hill C, Guarner F, Reid G, Gibson GR, Merenstein DJ, Pot B et al. The International Scientific Association for Probiotics and Prebiotics consensus statement on the scope and appropriate use of the term probiotic. Nat Rev Gastro Hepat. 2014;11(8):506-14. doi:10.1038/nrgastro.2014.66.

3. Barrett HL, Dekker Nitert M, Conwell LS, Callaway LK. Probiotics for preventing gestational diabetes. Cochrane Database Syst Rev. 2014(2):CD009951. doi:10.1002/14651858.CD009951.pub2.

4. Myhre R, Brantsaeter AL, Myking S, Gjessing HK, Sengpiel V, Meltzer HM et al. Intake of probiotic food and risk of spontaneous preterm delivery. Am J Clin Nutr. 2011;93(1):151-7. doi:10.3945/ajcn.110.004085.

5. Thum C, Cookson AL, Otter DE, Mcnabb WC, Hodgkinson AJ, Dyer J et al. Can Nutritional Modulation of Maternal Intestinal Microbiota Influence the Development of the Infant Gastrointestinal Tract? 2012;142(11):1921-8.

6. Edgar RC, Haas BJ, Clemente JC, Quince C, Knight R. UCHIME improves sensitivity and speed of chimera detection. Bioinformatics. 2011;27(16):2194-200. doi:10.1093/bioinformatics/btr381.

7. Haas BJ, Gevers D, Earl AM, Feldgarden M, Ward DV, Giannoukos G et al. Chimeric 16S rRNA sequence formation and detection in Sanger and 454-pyrosequenced PCR amplicons. Genome Res. 2011;21(3):494-504. doi:10.1101/gr.112730.110.

8. Edgar RC. UPARSE: highly accurate OTU sequences from microbial amplicon reads. Nat Methods. 2013;10(10):996-8. doi:10.1038/nmeth.2604.

9. Quast C, Pruesse E, Yilmaz P, Gerken J, Schweer T, Yarza P et al. The SILVA ribosomal RNA gene database project: improved data processing and web-based tools. Nucleic Acids Res. 2013;41(Database issue):D590-6. doi:10.1093/nar/gks1219.

10. Wang QJAEM. Naive Bayesian classifier for rapid assignment of rRNA sequences into the new bacterial taxonomy. 2007;73.

11. Edgar RC. MUSCLE: multiple sequence alignment with high accuracy and high throughput. Nucleic Acids Res. 2004;32(5):1792-7. doi:10.1093/nar/gkh340.

12. Satokari R, Gronroos T, Laitinen K, Salminen S, Isolauri E. Bifidobacterium and Lactobacillus DNA in the human placenta. Lett Appl Microbiol. 2009;48(1):8-12. doi:10.1111/j.1472-765X.2008.02475.x.

13. Jimenez E, Fernandez L, Marin ML, Martin R, Odriozola JM, Nueno-Palop C et al. Isolation of 
commensal bacteria from umbilical cord blood of healthy neonates born by cesarean section. Curr Microbiol. 2005;51(4):270-4. doi:10.1007/s00284-005-0020-3.

14. Steel JH, Malatos S, Kennea N, Edwards AD, Miles L, Duggan P et al. Bacteria and Inflammatory Cells in Fetal Membranes Do Not Always Cause Preterm Labor.57(3):404-11.

15. Aagaard K, Ma J, Antony KM, Ganu R, Petrosino J, Versalovic J. The placenta harbors a unique microbiome. Sci Transl Med. 2014;6(237):237ra65. doi:10.1126/scitranslmed.3008599.

16. Karlsson H, Larsson P, Wold AE, Rudin A. Pattern of cytokine responses to gram-positive and gram-negative commensal bacteria is profoundly changed when monocytes differentiate into dendritic cells. Infect Immun. 2004;72(5):2671-8. doi:10.1128/Iai.72.5.2671-2678.2004.

17. Cukrowska B, Lodinova-Zadnikova R, Enders C, Sonnenborn U, Schulze J, Tlaskalova-Hogenova H. Specific proliferative and antibody responses of premature infants to intestinal colonization with nonpathogenic probiotic E-coli strain Nissle 1917. Scand J Immunol. 2002;55(2):204-9. doi:DOI 10.1046/j.1365-3083.2002.01005.x.

18. Wall R, Cryan JF, Ross RP, Fitzgerald GF, Dinan TG, Stanton C. Bacterial Neuroactive Compounds Produced by Psychobiotics. Adv Exp Med Biol. 2014;817:221-39. doi:10.1007/978-1-4939-0897-4_10. 19. Liang S, Wang T, Hu X, Luo J, Li W, Wu X et al. Administration of Lactobacillus Helveticus Ns8 Improves Behavioral, Cognitive, and Biochemical Aberrations Caused by Chronic Restraint Stress. Neuroscience. 2015;310:561-77. doi:10.1016/j.neuroscience.2015.09.033.

20. Koren O, Goodrich JK, Cullender TC, Spor A, Laitinen K, Backhed HK et al. Host remodeling of the gut microbiome and metabolic changes during pregnancy. Cell. 2012;150(3):470-80. doi:10.1016/j.cell.2012.07.008.

21. Perez PF, Dore J, Leclerc M, Levenez F, Benyacoub J, Serrant P et al. Bacterial imprinting of the neonatal immune system: lessons from maternal cells? Pediatrics. 2007;119(3):e724-32. doi:10.1542/peds.2006-1649.

22. Katz J, Chegini N, Shiverick KT, Lamont RJ. Localization of P. gingivalis in preterm delivery placenta. J Dent Res. 2009;88(6):575-8. doi:10.1177/0022034509338032.

23. Ogbonnaya ES, Clarke G, Shanahan F, Dinan TG, Cryan JF, Psychiatry OOLJB. Adult Hippocampal Neurogenesis Is Regulated by the Microbiome. 2015;78(4):e7-e9.

24. XU Zhen-guo, CAI Yu-hua, LIU Xiu-shu, FAN Gao-fu, DAI Yin. Research progress and application prospect of Bifidobacterium. Chin J Biologicals. 2017;30(002):215-20.

25. Hill C, Guarner F, Reid G, Gibson GR, Merenstein DJ, Pot B et al. Expert consensus document: The International Scientific Association for Probiotics and Prebiotics consensus statement on the scope and appropriate use of the term probiotic. 2014;11(8):506-14.

26. Plovier H, Cani PD. Microbial Impact on Host Metabolism: Opportunities for Novel Treatments of Nutritional Disorders? Microbiol Spectr. 2017;5(3). doi:10.1128/microbiolspec.BAD-0002-2016.

27. Zang Kaili, J.Y., SUN Yong, YAN Yali, CHEN Qingsen, ZHAO Linsen, ZHAO Pei1, CUI Wenjing and M.X. , Probiotics Modulate the Structure and Abundance of Gut Mircrobiota in Populations with Intestinal Diseases. Food Science 2018;39(13): p. 133-143.

28. ZHOU Meng-jia, N.X.-q., ZENG Dong, ZHANG Lu, and T. Teng, Effects of Probiotic Bacteria on Serum Antioxidant Capacity and Intestinal Flora of Mice Gavaged with Alcohol. Food Science 2013;34(23): p. 309-312.

29. Hoyme UB, Saling E. Efficient prematurity prevention is possible by $\mathrm{pH}$-self measurement and immediate therapy of threatening ascending infection. European Journal of Obstetrics Gynecology and Reproductive Biology. 2004;115(2):148-53. doi:10.1016/j.ejogrb.2004.02.038. 
30. Dugoua JJ, Machado M, Zhu X, Chen X, Koren G, Einarson TR. Probiotic safety in pregnancy: a systematic review and meta-analysis of randomized controlled trials of Lactobacillus, Bifidobacterium, and Saccharomyces spp. J Obstet Gynaecol Can. 2009;31(6):542-52. doi:10.1016/S1701-2163(16)34218-9. 
Figures

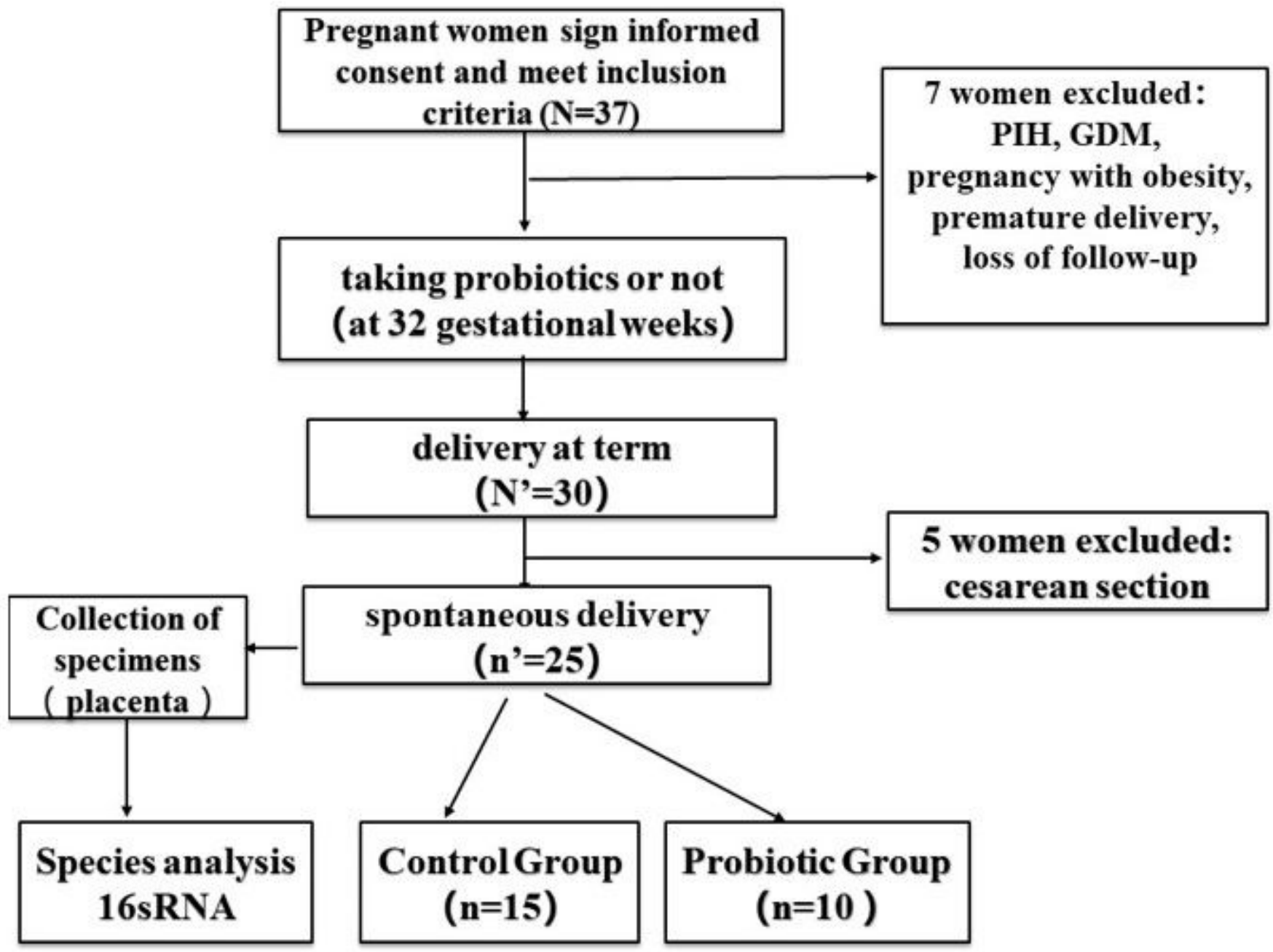

Figure 1

Technical flow chart for screening research subjects 


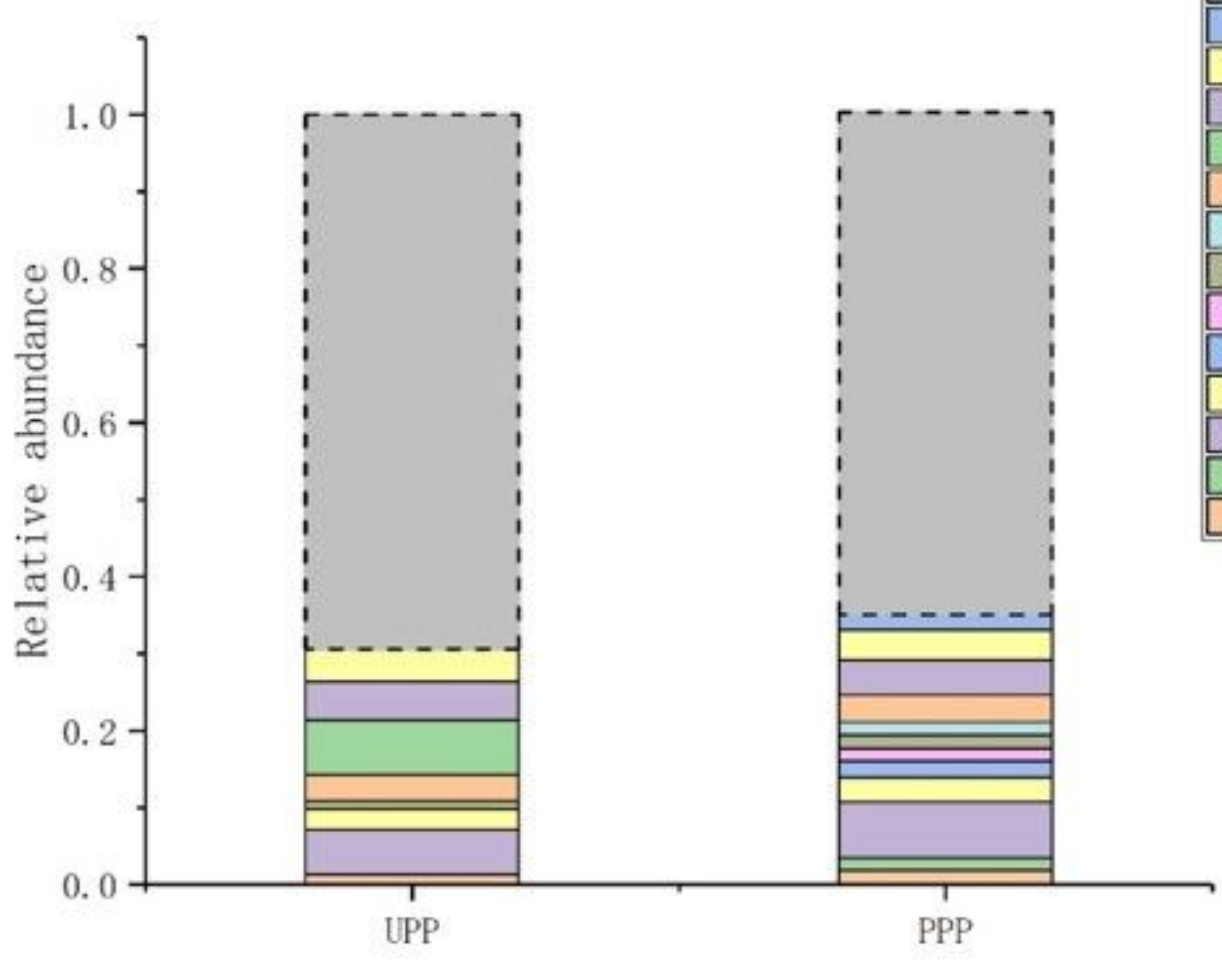

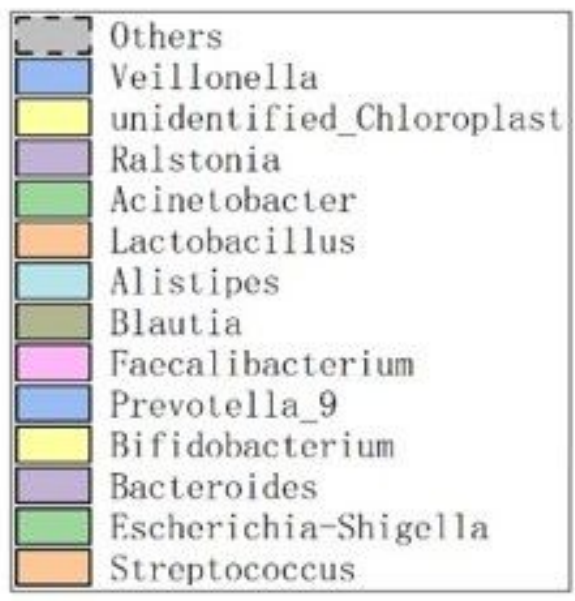

\section{Figure 2}

Relative abundances of operational taxonomic units (OTUs) at genus level. (Note: UPP: control group; PPP: probiotic group; the relative abundances of OTUs accounting for above 0.01 in the placental microorganisms are shown for each group using the 16S Ribosomal RNA gene deep sequencing analysis. OTUs representing less than 0.01 are grouped as "others".)

(a)

(b)

\section{Cladogram}
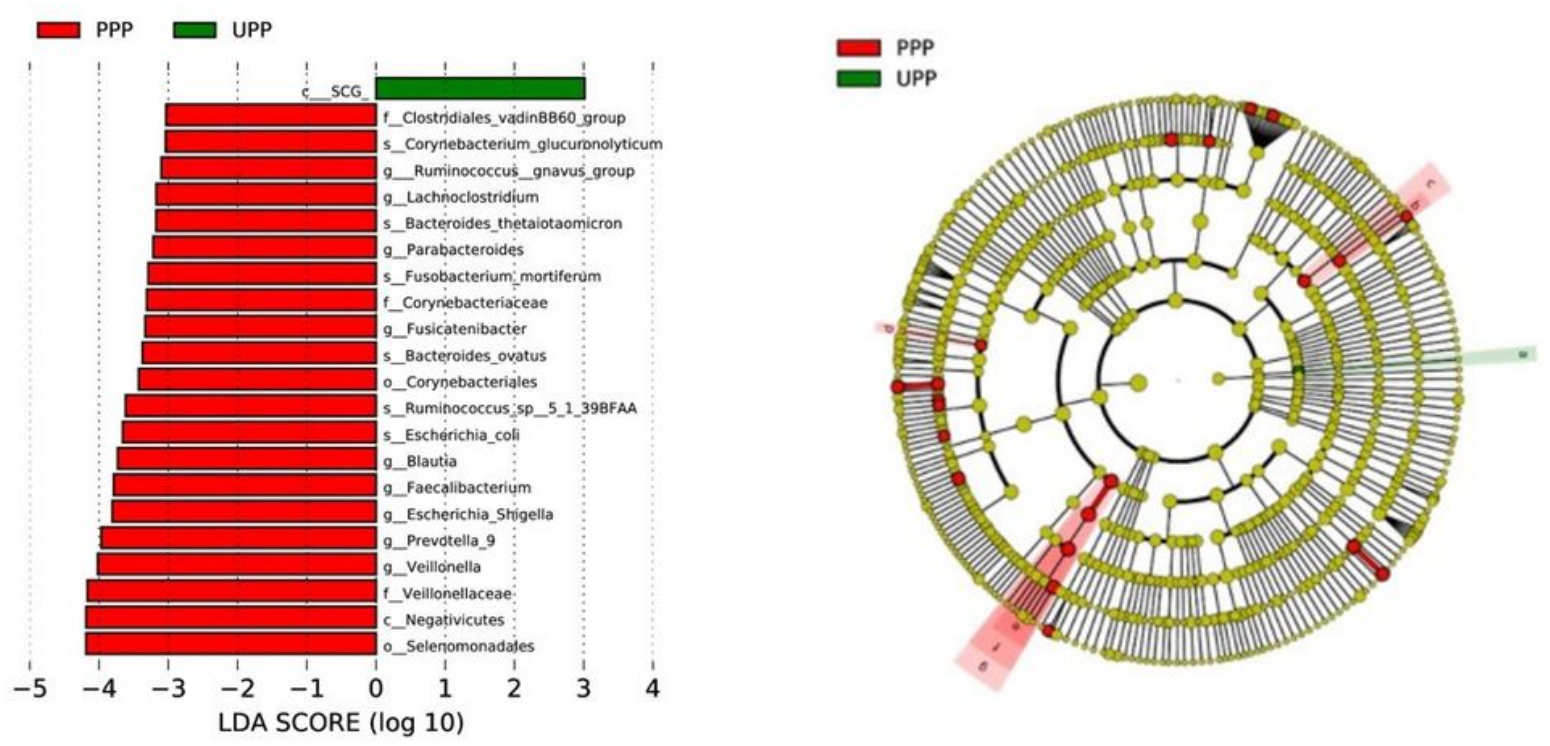


\section{Figure 3}

LEfSe analysis of full-term placental microbiome in two groups. (a) Differences in key OTUs identified as differentiating between probiotic and control group visualized using a LDA columnar distribution. (b) Bacterial taxa that were differentially abundant in probiotic and control group visualized using a cladogram generated from LEfSe analysis. (Note a: UPP: control group; PPP: probiotic group; The abscissa represents LDA score greater than 3 , the ordinate represents the species of the flora, and the length of the histogram represents the influence of the differential flora. The LDA threshold is set to 3 (Log LDA > 3.00). If the Group, LDA, and P values are all empty, it means that the species has no difference between the group. In the above picture. c:class, o:order, f: family, g: genus, s:species. Note b: UPP: control group; PPP: probiotic group; Red regions represent the PPP group, and green regions represent the UPP group. The red node indicates the microbial group that plays an important role in the red group, the green node indicates the microbial group that plays an important role in the green group, and the yellow indicates the species that have no significant difference between the groups.) 


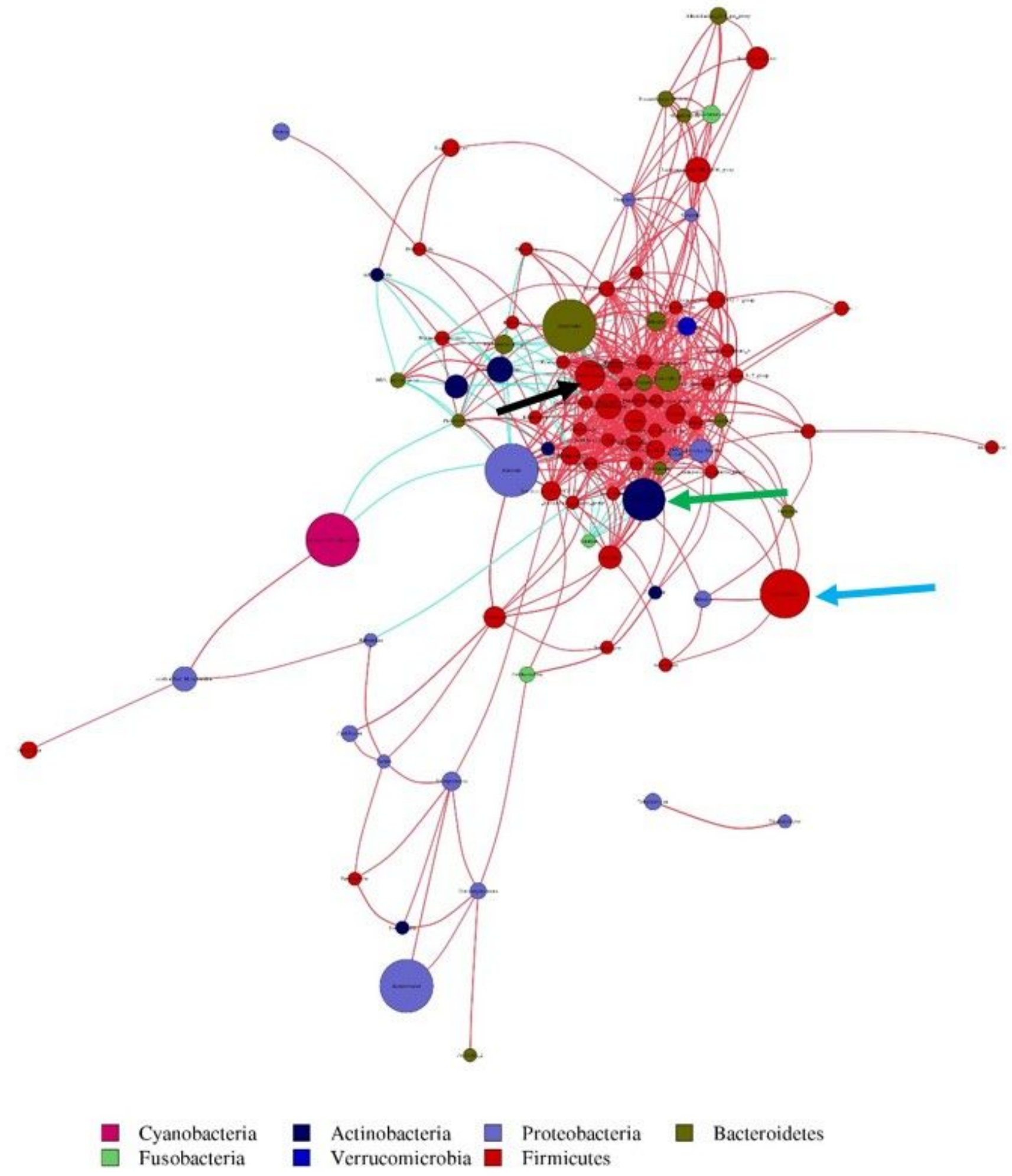

\section{Figure 4}

The interaction network structure of placental microbiome in control group (UPP group). Note: The Lactobacillus, Bifidobacterium, and Streptococcus were pointed by blue, green and black arrow respectively. The nodes in the above figure are identified by different colors, representing each dominant genus. The line between the nodes indicates a correlation between the two genera, the red line indicates a positive correlation, the blue line indicates a negative correlation, and the thickness of the line indicates 
the intensity of correlation. The node size represents the content of the OTUs, and the dots of the same color represent the genus classified into the same phylum. The more connections through a node, the more associations the genus has with other members of the genus.

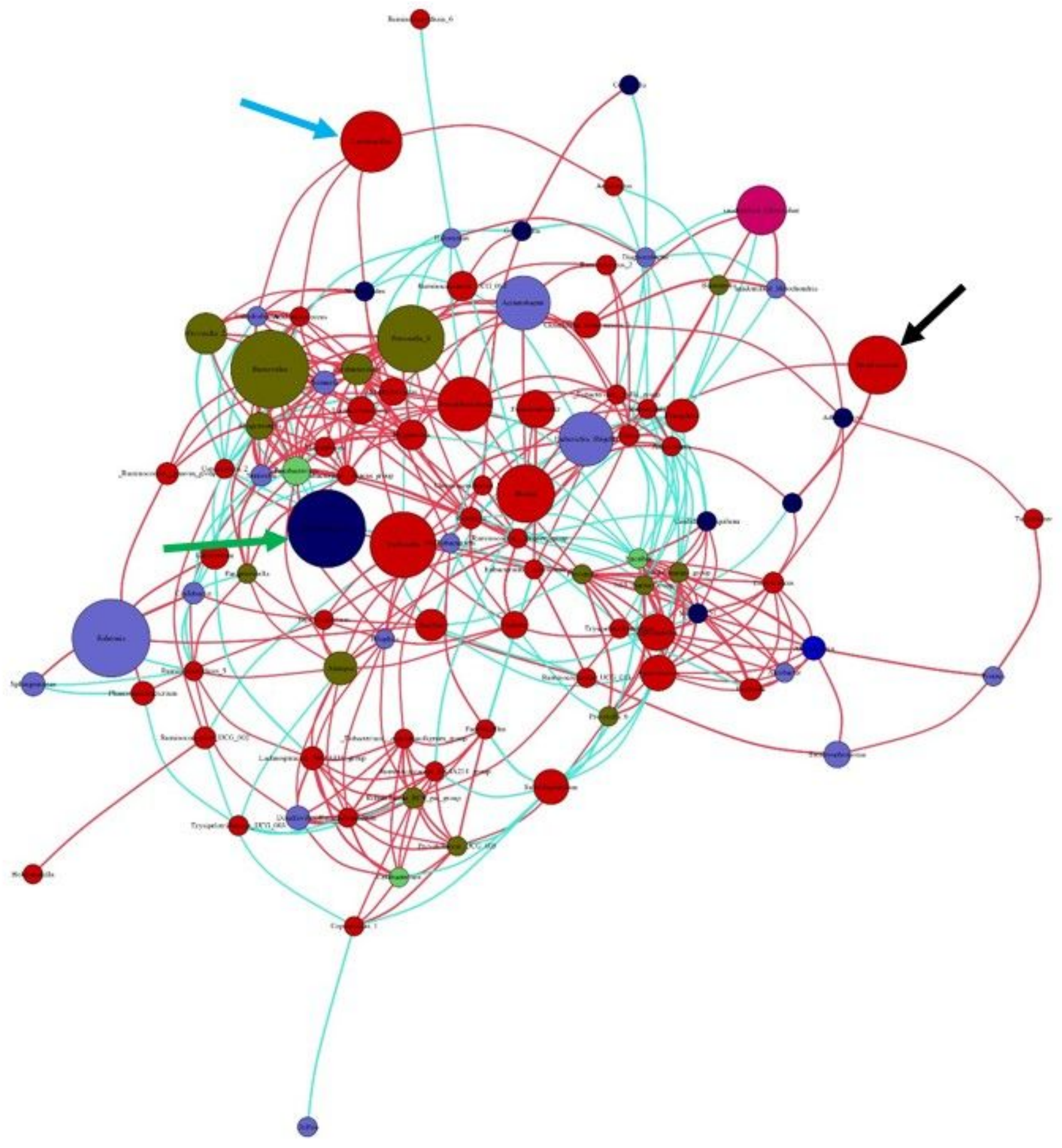

Cyanobacteria Fusobacteria
Actinobacteria Verrucomicrobia
Bacteroidetes Firmicutes
Proteobacteria

\section{Figure 5}

The interaction network structure of placental microbiome in probiotic group (PPP group). Note: The Lactobacillus, Bifidobacterium, and Streptococcus were pointed by blue, green and black arrow 
respectively. The nodes represent each dominant genus. The line between the nodes indicates a correlation between the two genera, where the red line indicates a positive correlation, and the blue line indicates a negative correlation, and the thickness of the line indicates the intensity of correlation. The node size represents the content of the OTUs, and the dots of the same color represent that the genus is classified into the same phylum. The more connections through a node, the more associations the genus has with other members of the genus. 